\title{
miR-331-3p inhibits proliferation and promotes apoptosis by targeting HER2 through the PI3K/Akt and ERK1/2 pathways in colorectal cancer
}

\author{
DONGLI ZHAO ${ }^{1}$, YANXIA SUI $^{2}$ and XIAOQIANG ZHENG ${ }^{1}$ \\ Departments of ${ }^{1}$ Radiotherapy and ${ }^{2}$ Pathology, The First Affiliated Hospital of \\ Xi'an Jiaotong University, Xi'an, Shaanxi 710061, P.R. China
}

Received August 8, 2015; Accepted September 26, 2015

DOI: $10.3892 /$ or.2015.4450

\begin{abstract}
MicroRNAs (miRNAs) regulate cell proliferation, apoptosis and carcinogenesis by targeting related mRNAs in different types of cancer. miR-331-3p has been found to regulate the development and progression of various types of cancer cells. However, little research has been conducted on the role of miR-331-3p in colorectal cancer (CRC). The present study aimed to explore the function of miR-331-3p in CRC. We found that miR-331-3p was significantly downregulated in CRC tissues and cells compared to the level in healthy colon tissues and cells. Overexpression of miR-331-3p by transfection with pre-miR-331-3p inhibited cell proliferation, promoted apoptosis and activated caspase-3. Furthermore, the protein expression level of apoptosis-related protein Bcl-2 was downregulated and Bax was upregulated by pre-miR-331-3p. Downregulation of the expression of miR-331-3p by transfection with AS-miR-331-3p had the opposite effect. Moreover, we found that HER2 was overexpressed in the CRC cell lines, and the expression level of HER2 was negatively regulated by miR-331-3p. Additionally, knockdown of HER2 inhibited cell proliferation and phosphorylation of Akt and ERK1/2 induced by AS-miR-331-3p. Overall, we identified that miR-331-3p is underexpressed in CRC and contributes to cell growth regulation by targeting HER 2 through activating the PI3K/Akt and ERK1/2 signaling pathways.
\end{abstract}

\section{Introduction}

Colorectal cancer (CRC) is one of the most common cancers in the world and the third leading cause of cancer-related death

Correspondence to: Dr Yanxia Sui, Department of Pathology, The First Affiliated Hospital of Xi'an Jiaotong University, 277 Yanta West Road, Xi'an, Shaanxi 710061, P.R. China

E-mail: yanxiasui@126.com

Abbreviations: miRNAs, microRNAs; CRC, colorectal cancer; 3'UTR, 3' untranslated region; EGFR, epidermal growth factor receptor

Key words: colorectal cancer, HER2, miR-331-3p, ErbB2 in both males and females (1). It was estimated that there were more than 1.4 million CRC cases and 693,900 deaths in 2012 worldwide (2). Smoking, red/processed meat consumption, obesity and excessive alcohol consumption are the modifiable risk factors for CRC (3). With a reduction in smoking and other modifiable risk factors, along with CRC screening and research into the molecular pathological epidemiology of CRC, decreased CRC mortality rates have been observed $(4,5)$. However, the death rate is still high, and the molecular pathological epidemiology of CRC is not completely understood, thus further extensive investigations are required.

MicroRNAs (miRNAs) are 18-23-nucleotide small RNAs that function as negative regulators of gene expression through binding the 3' untranslated region (3'UTR) of mRNAs (6). Since evidence of the involvement of miR-15 and miR-16 in leukemia was initially reported, researchers have investigated the important roles of miRNAs in cancer $(7,8)$. Subsequently, more and more miRNAs have been found to be involved in the development of human tumors (9). miRNAs can regulate cell proliferation, apoptosis and carcinogenesis by targeting related mRNAs in cancer (10). In CRC, eight miRNAs, including miR-25, miR-345, miR-7 and miR-331-3p, have been found to be significantly and differentially expressed when compared to the levels in healthy tissues (11).

miR-331-3p is a member of the miR-331 family, located on $12 \mathrm{q} 22 \mathrm{n}$, and has been shown to be a tumor-suppressor miRNA in the prostate (12). Aberrant expression of miR-331-3p has been observed and found to be associated with proliferation and migration in lymphocytic leukemia (13), lung cancer (14), glioblastoma (15), gastric cancer (16) and prostate cancer (17). However, the role of miR-331-3p in the tumorigenesis of CRC remains unknown (18).

The human epidermal growth factor receptor (EGFR) family, which consists of EGFR (HER1 or ErbB1), HER2 (HER2/neu or ErbB2), HER3 (ErbB3) and HER4 (ErbB4), plays an important role in regulating cell proliferation, survival, and differentiation in cancers $(19,20)$. HER2 is an important regulator of EGFR family signaling $(21,22)$. Overexpression of HER 2 is found in $\sim 30 \%$ of all breast cancers and is frequently associated with poor prognosis, greater invasiveness and higher apoptotic resistance potential (23-26). It has been found that HER2 is a key factor in CRC, and cytoplasmic HER2 is 
overexpressed in almost $30 \%$ of CRC patients (27). However, the prognostic value of HER-2 expression remains controversial in CRC. Thus, the present study was designed to validate the potential function of miR-331-3p in CRC.

\section{Materials and methods}

Human tissue samples. Human colon cancer tissue samples and normal adjacent colon tissues were obtained from 29 colon cancer patients before any therapeutic intervention at the First Affiliated Hospital of Xi'an Jiaotong University. All patients provided informed written consent. Samples were selected and stored in liquid nitrogen immediately after surgical resection. The study was approved by the Human Research Ethics Committee of the hospital.

Cell culture. Human colon cancer cell lines HCT-116, LoVo, HT-29, SW480, DLD-1 and Caco2 and the human normal colon epithelial cell line CRL-1831 (American Type Culture Collection, ATCC, Manassas, VA, USA) were grown in RPMI1640 medium containing 5\% fetal bovine serum (FBS) (both from Gibco-BRL, Gaithersburg, MD, USA), $1 \%$ penicillin and streptomycin (Sigma-Aldrich, Castle Hill, NSW, Australia), and maintained in a humidified incubator at $37^{\circ} \mathrm{C}$ with $5 \% \mathrm{CO}_{2}$.

Real-time quantitative polymerase chain reaction ( $R T-q P C R)$. MicroRNAs were isolated from the tissue samples and cells using the miRNeasy Mini kit (Qiagen, Valencia, CA, USA). One Step PrimeScript miRNA cDNA Synthesis kit (Takara Biotechnology, Dalian, China) was used to synthesis cDNA. Total RNA was extracted using TRIzol reagent (Invitrogen, Carlsbad, CA, USA) and reverse-transcribed into cDNA using M-MLV reverse transcriptase (Clontech, Palo Alto, CA, USA) according to the standard protocol. RT-qPCR was performed using SYBR Green qPCR Master Mix (Thermo Fisher, Shanghai, China). U6 (RiBoBio, Guangzhou, China) or $\beta$-actin was used as the normalizer for miRNA or mRNA, respectively. The data obtained were assessed using the $2^{-\Delta \Delta \mathrm{Ct}}$ method and evaluated by statistical analysis as described previously (28).

Plasmid vectors and transfection. HCT-116 cells were transfected with the miR-331-3p precursor (pre-miR-331-3p) or negative control RNA oligonucleotides (pre-miR-control) (both from Ambion Corporation, Austin, TX, USA); the miRNA inhibitor of miR-331-3p (AS-miR-331-3p) or the negative control (AS-miR-control); and HER2-siRNA (29) (sense, 5'-GGUGAAGGUGCUUGGAUCUUU-3' and antisense, 5'-AGAUCCAAGCACCUUCACCUU-3') or control siRNA using Lipofectamine 2000 according to the manufacturer's procedure (Invitrogen) and cultured for $48 \mathrm{~h}$. The expression levels of miR-331-3p and HER2 in the HCT-116 cell line were assayed by RT-qPCR $48 \mathrm{~h}$ after transfection.

Caspase-3 activity assay. A caspase-3 assay kit (Abcam, Cambridge, MA, USA) was used to measure the enzymatic activity of caspase-3. HCT-116 cells transfected with pre-miR-331-3p or AS-miR-331-3p were seeded in a 24-well plate and cultured for $48 \mathrm{~h}$. The cells were then harvested and the enzymatic activity of caspase- 3 was detected according to the manufacturer's protocol.
Cell proliferation assay. HCT-116 cells transfected with pre-miR-331-3p or AS-miR-331-3p or HER2-siRNA or HER2siRNA and AS-miR-331-3p were seeded in a 96-well plate. Twenty-four, 48, 72 and $96 \mathrm{~h}$ later, the medium was replaced by fresh medium, and $20 \mu \mathrm{l}$ MTT $(5 \mathrm{mg} / \mathrm{ml})$ was added in each well and incubated for $4 \mathrm{~h}$. Then, the medium was removed, and $200 \mu \mathrm{l}$ DMSO was added. The OD490 was read after shaking for $10 \mathrm{~min}$ to fully dissolve the crystals.

Cell apoptosis assay. HCT-116 cells transfected with pre-miR-331-3p or AS-miR-331-3p or HER2-siRNA or HER2siRNA and AS-miR-331-3p were seeded in 24-well plates and cultured for $48 \mathrm{~h}$. Cells were harvested and the apoptotic cells were evaluated using the FITC-Annexin V apoptosis detection kit (BD Biosciences, Piscataway, NJ, USA) according to the manufacturer's instructions.

Western blot analysis. HCT-116 cells transfected with pre-miR-331-3p or AS-miR-331-3p were seeded in a 6-well plate and cultured for $48 \mathrm{~h}$. Cells were harvested and the total protein was extracted using mammalian protein extraction reagent (Pierce, Rockford, IL, USA) supplemented with a protease inhibitor cocktail (Sigma, St. Louis, MO, USA). After measuring the concentration, the proteins were separated using $10 \%$ SDS-PAGE and then transferred onto a nitrocellulose membrane (Bio-Rad, Hercules, CA, USA). The membranes were blocked in 5\% (v/v) dried milk and incubated with anti-HER2 (Abcam), anti-Bax, anti-Bcl-2, anti-Akt, anti-p-Akt, anti-ERK1/2 and anti-p-ERK1/2 (Santa Cruz Biotechnology, Santa Cruz, CA, USA ) at $4^{\circ} \mathrm{C}$ overnight. The secondary HRP-conjugated goat anti-mouse IgG antibody (Santa Cruz Biotechnology) was incubated for $1 \mathrm{~h}$. $\beta$-actin (Cell Signaling Technology, Danvers, MA, USA) was used as the reference protein.

Luciferase activity assay. The human HER 2 wild-type 3'UTR containing the miR-331-3p binding site and mutated HER2-3'UTR sequence (18) were constructed into the pGL3 luciferase reporter plasmid (Promega, Madison, WI, USA). HCT-116 cells were plated in 24-well plates and co-transfected with $10 \mathrm{nM}$ of either pre-miR 331-3p or the pre-miR-control, and $500 \mathrm{ng}$ of pGL3-HER2 or pGL3-mutHER2 according to the manufacturers' protocols for the use of Lipofectamine (Invitrogen) and the luciferase assay kit (Promega) (16). Cells were collected and cell lysates were assayed for luciferase activity using a dual-luciferase reporter assay kit (Promega) $48 \mathrm{~h}$ after transfection.

Statistical analysis. Results are presented as the mean \pm standard deviation. One-way analysis of variance and the Student's t-test were used to analyze differences between two groups. $\mathrm{P}<0.05$ was considered to indicate a statistically significant difference.

\section{Results}

miR-331-3p is downregulated in human colon cancer tissues and cells. To explore the role of miR-331-3p in human colon cancer, the expression level of miR-331-3p in human colon cancer tissues and cells was measured using RT-qPCR. 

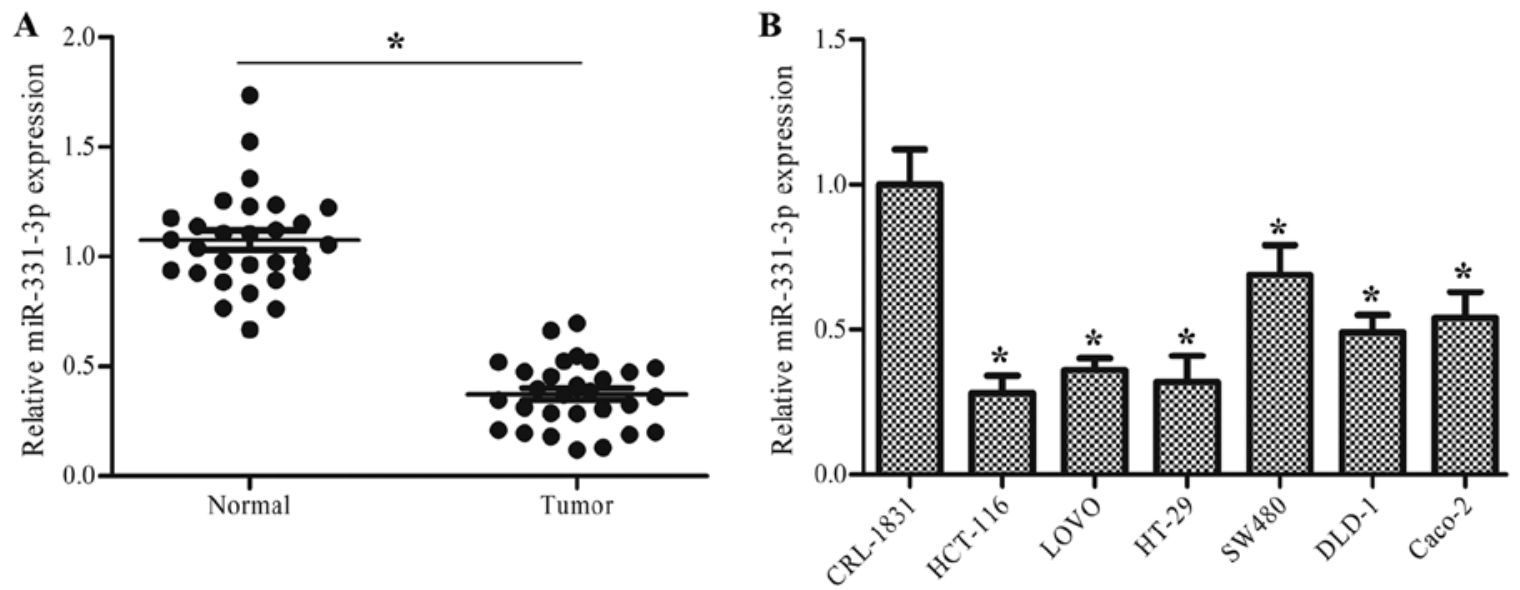

Figure 1. miR-331-3p is downregulated in the colon cancer tissues and cell lines. (A) RT-qPCR analysis of miR-331-3p expression in colon cancer tissues from 29 CRC patients and normal adjacent colon tissues. The values of each dot represent the ratios of miR-331-3p expression in CRC tissues and adjacent colon tissues. (B) RT-qPCR analysis of miR-331-3p expression in CRC cell lines and the normal colon epithelial cell line CRL-1831. The values of each column represent the ratio of miR-331-3p expression in the CRC cell line and the level in the normal colon epithelial cell line. The bars in the histogram represent the mean \pm standard deviation of three independent experiments. " $\mathrm{P}<0.05$ vs. normal or CRL-1831.
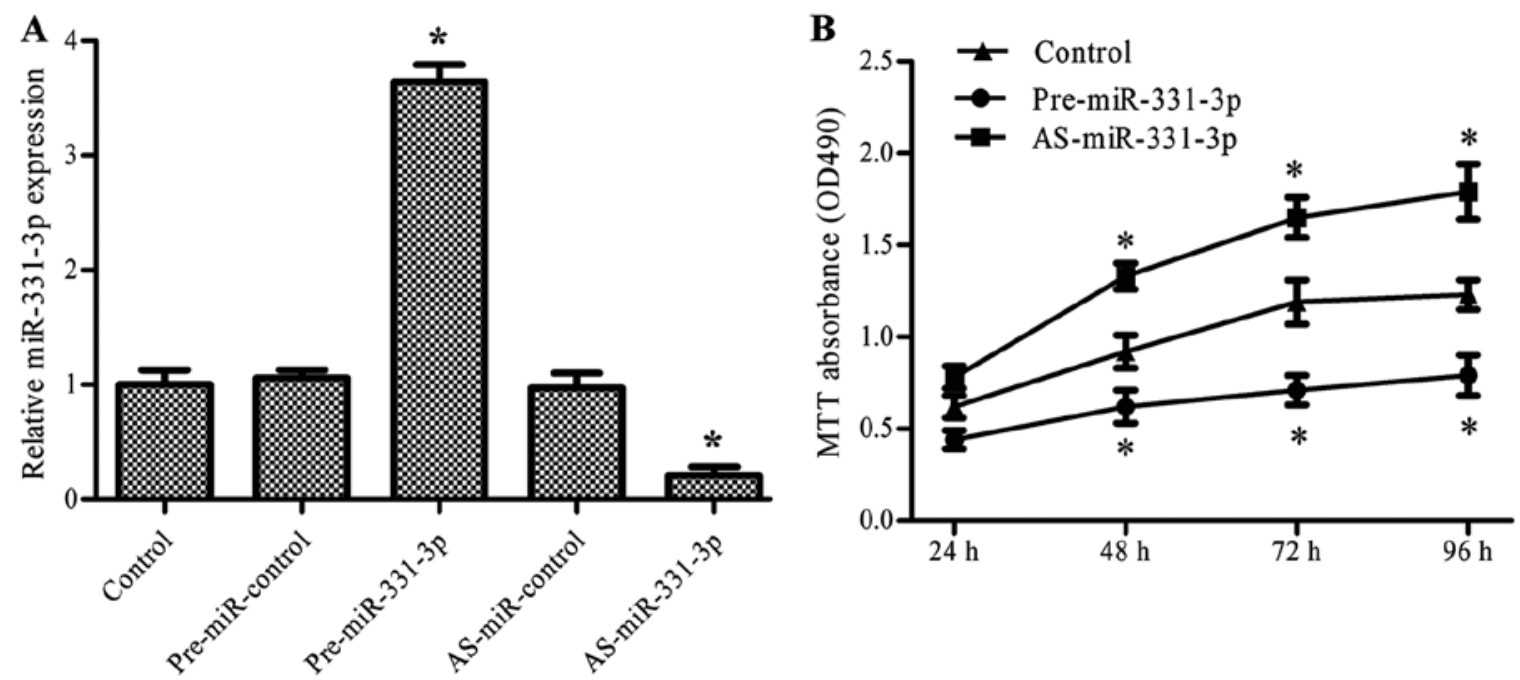

Figure 2. miR-331-3p inhibits colon cancer cell proliferation. (A) RT-PCR analysis of miR-331-3p expression in the HCT-116 cells transfected with pre-miR-331-3p or AS-miR-331-3p for $48 \mathrm{~h}$. Bars in the histogram represent the mean \pm standard deviation of three independent experiments. "P<0.05 vs. the control. (B) Cell proliferation was detected by MTT assay after the HCT-116 cells were transfected with pre-miR-331-3p or AS-miR-331-3p for 24, 48, 72 and $96 \mathrm{~h}$. Values represent the mean \pm standard deviation of three independent experiments. ${ }^{*} \mathrm{P}<0.05$ vs. control.

The results showed that the expression level of miR-331-3p was significantly downregulated in the colon cancer tissues compared to this level in the normal tissues $(\mathrm{P}<0.05$, Fig. 1A). Compared with the normal colon epithelial cell line CRL-1831, the expression level of miR-331-3p was significantly lower in all of the colon cancer cell lines, including HCT-116, LoVo, HT-29, SW480, DLD-1 and Caco2 (P<0.05, Fig. 1B).

miR-331-3p inhibits colon cancer cell proliferation. To verify the effect of miR-331-3p on the growth of colon cancer, we examined the impact of miR-331-3p overexpression and suppression on the proliferation of colon cancer cells. HCT-116 cells were transfected with pre-miR-331-3p or AS-miR-331-3p and cultured for various time periods. The MTT assay was then used to evaluate the proliferation of the colon cancer cells. The results revealed that pre-miR-331-3p significantly upregulated and AS-miR-331-3p downregulated the expression of miR-331-3p ( $\mathrm{P}<0.05$, Fig. 2A). The MTT assay showed that pre-miR-331-3p inhibited and AS-miR-331-3p promoted the proliferation of the HCT-116 cells in a time-dependent manner ( $\mathrm{P}<0.05$, Fig. 2B).

miR-331-3p promotes the apoptosis of the colon cancer cells. To further confirm the effect of miR-331-3p on the tumorigenesis of colon cancer, the apoptotic ratio of the HCT-116 cells transfected with pre-miR-331-3p or AS-miR331-3p was examined by FITC-Annexin V staining. The results show that overexpression of miR-331-3p significantly promoted apoptosis while suppression of miR-331-3p inhibited apoptosis $(\mathrm{P}<0.05$, Fig. 3A). To further study the effect 

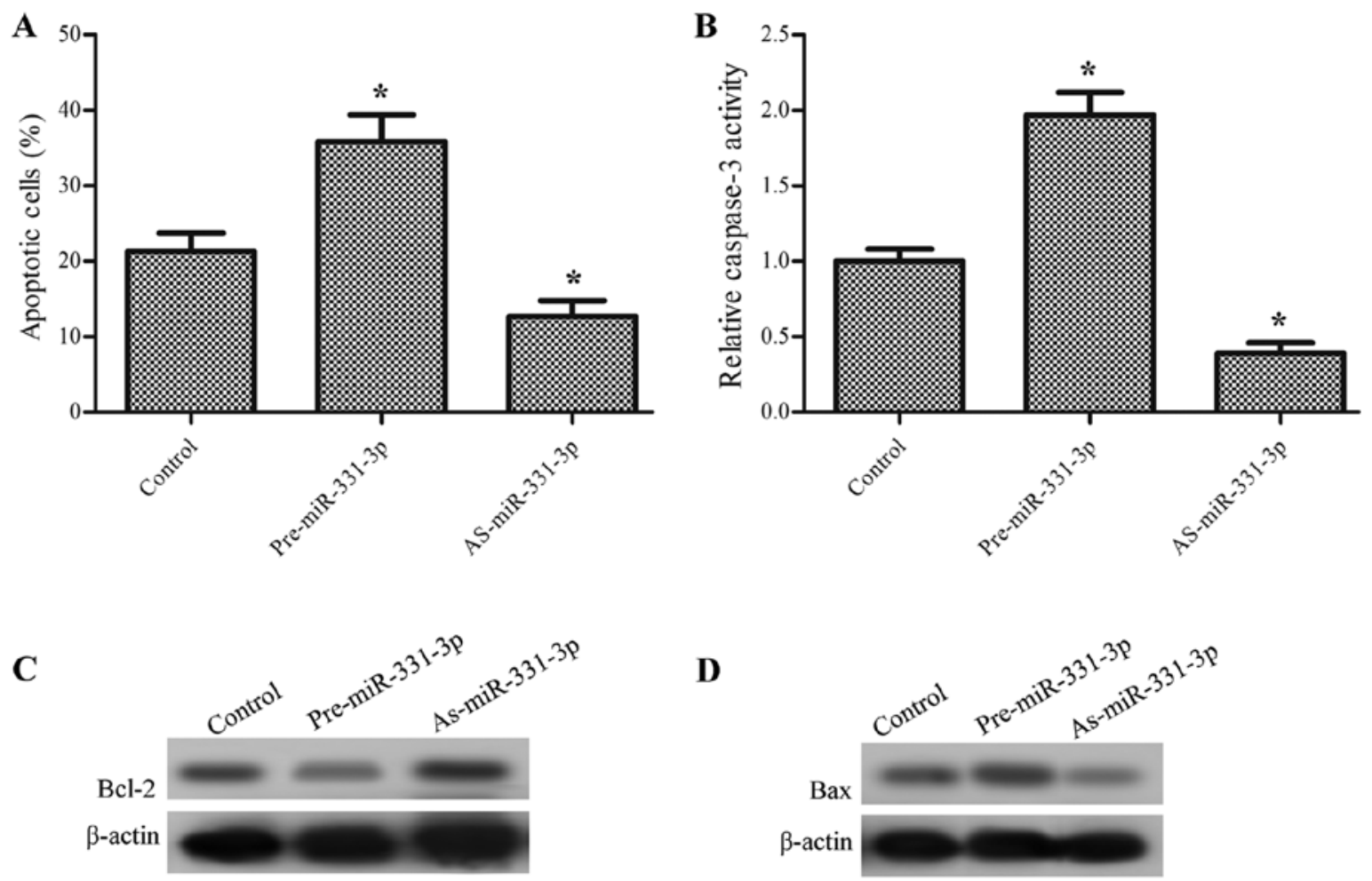

D
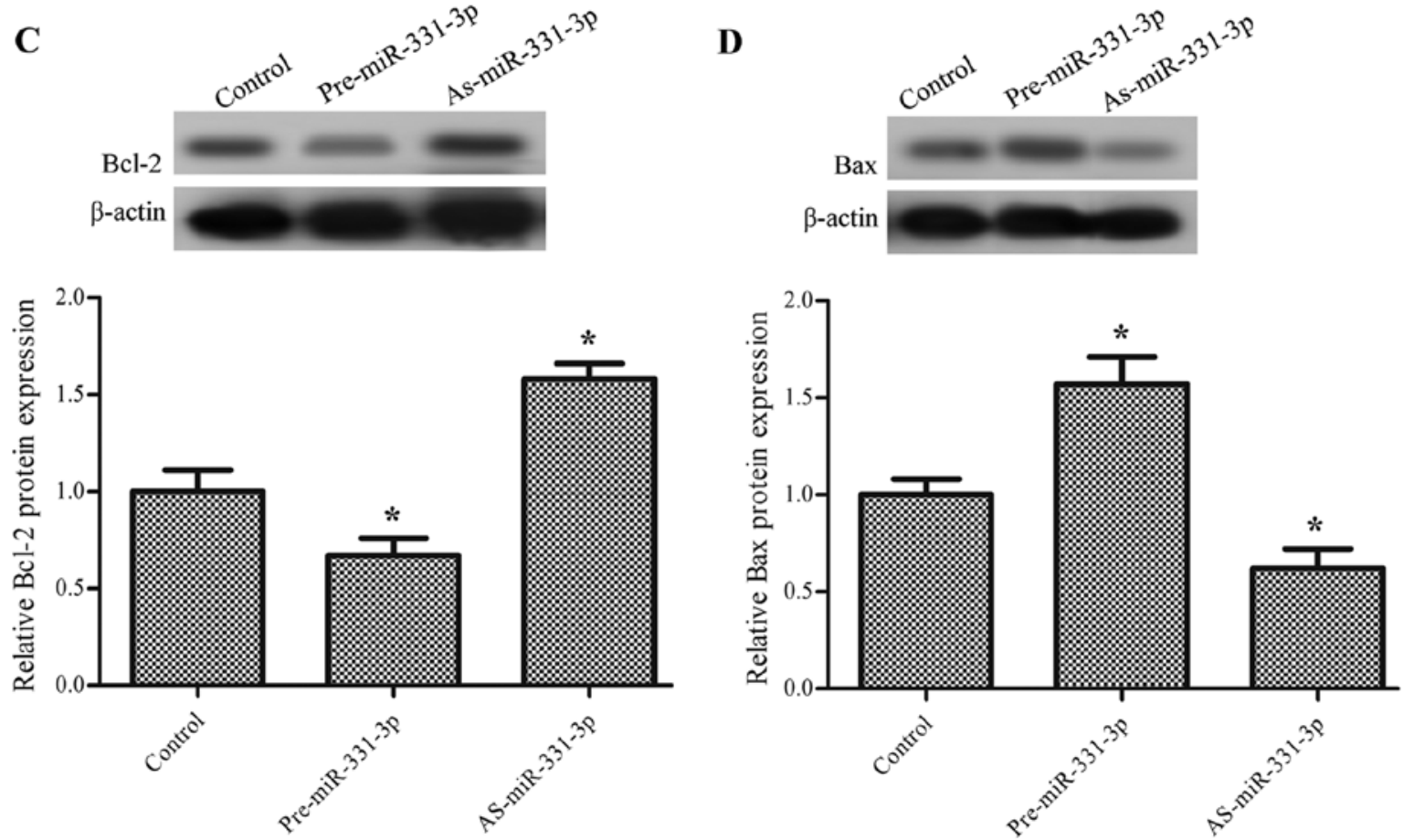

Figure 3. miR-331-3p promotes the apoptosis of colon cancer cells. HCT-116 cells were transfected with pre-miR-331-3p or AS-miR-331-3p for 48 h. (A) Cell apoptosis was detected by flow cytometry. (B) The enzymatic activity of caspase-3 was assessed using the caspase-3 assay kit. (C) Western blot analysis of the protein expression level of Bcl-2. (D) Western blot analysis of the protein expression level of Bax. Bars in the histograms represent the mean \pm standard deviation of three independent experiments. ${ }^{*} \mathrm{P}<0.05$ vs. the control.

of miR-331-3p on colon cancer cell apoptosis, the activity of caspase-3 was also determined. As expected, pre-miR-331-3p significantly increased the activity of caspase-3 and AS-miR331-3p had the opposite effect ( $\mathrm{P}<0.05$, Fig. 3B). Additionally, the protein expression levels of the apoptosis-related proteins Bcl-2 and Bax were also measured; the results demonstrated that pre-miR-331-3p decreased the protein expression level of Bcl-2 and increased the protein expression level of Bax, while AS-miR-331-3p increased the protein expression level of Bcl-2 and decreased the protein expression level of Bax $(\mathrm{P}<0.05$, Fig. $3 \mathrm{C}$ and $\mathrm{D})$.

miR-331-3p directly downregulates the expression of HER2 in colon cancer cells. The expression level of HER2 in the human colon cancer cells was significantly higher than that in the normal cells, which was inversely related to the expression of miR-331-3p ( $\mathrm{P}<0.05$, Fig. $4 \mathrm{~A}$ and B). Next, we explored the relationship between miR-331-3p and HER2 by transfecting cells with pre-miR-331-3p and AS-miR331-3p. The western blot analysis shows that pre-miR-331-3p significantly downregulated and AS-miR-331-3p significantly upregulated the protein expression level of HER2 in the HCT-116 cells ( $\mathrm{P}<0.05$, Fig. 4C), suggesting that miR-331-3p downregulates the expression of HER2 in colon cancer cells. To examine whether HER2 is a direct target of miR331-3p in colon cancer cells, the human HER2 wild-type 3'UTR containing the miR-331-3p binding site and mutated HER2 3'UTR sequence were cloned into modified pGL-3 
$\mathbf{A}$
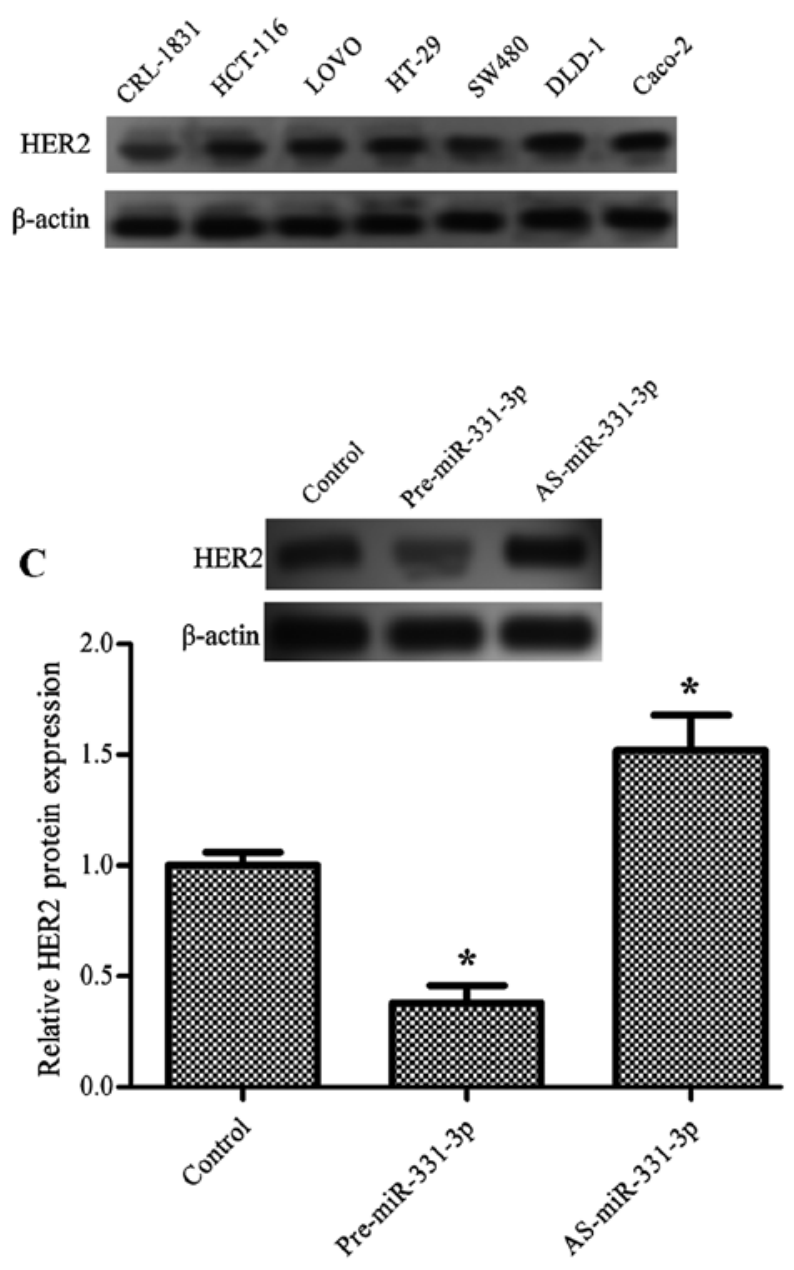

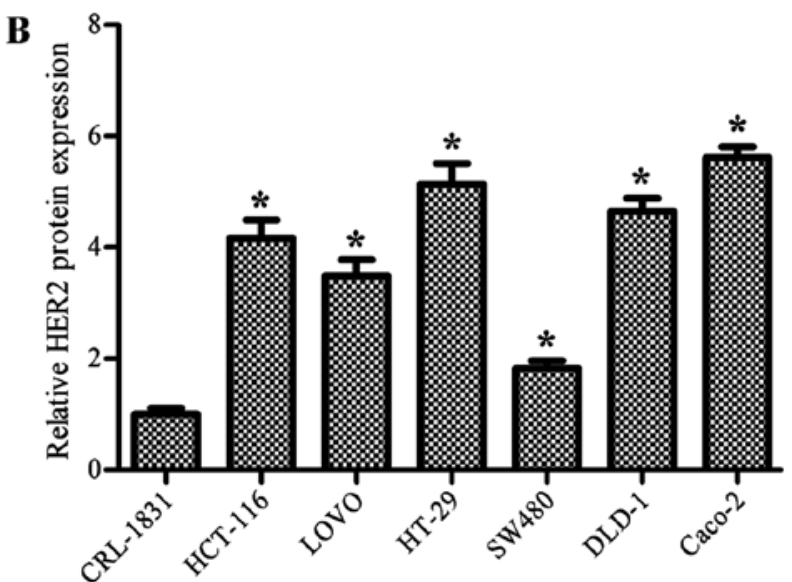

D

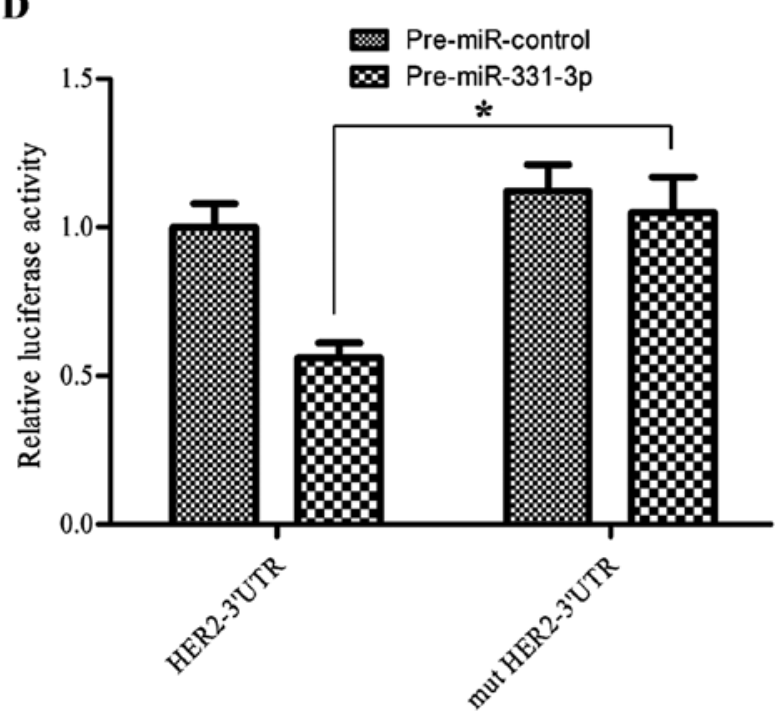

Figure 4. miR-331-3p downregulates the expression of HER2 in colon cancer cells. (A) Representative western blot of the expression of HER2 in the various CRC cell lines. (B) The quantification of the protein expression level of HER2 was normalized to $\beta$-actin in the CRC cell lines. Bars in the histogram represent the mean \pm standard deviation of three independent experiments. $\mathrm{P}<0.05$ vs. the CRL-1831 cell line. (C) Western blot analysis of the protein expression levels of HER2 in the HCT-116 cells transfected with pre-miR-331-3p or AS-miR-331-3p. Bars in the histogram represent the mean \pm standard deviation of three independent experiments. " $\mathrm{P}<0.05$ vs. the control. (D) Luciferase assay of HCT-116 cells transfected with pre-miR 331-3p or AS-miR-331-3p and pGL3-HER2 or pGL3-mutHER2. Bars in the histogram represent the mean \pm standard deviation of three independent experiments. ${ }^{*} \mathrm{P}<0.05 \mathrm{vs}$. pre-miR-control.

luciferase reporter vectors, which were co-transfected into the HCT-116 cells with pre-miR-331-3p and AS-miR-331-3p. The results showed that miR-331-3p overexpression significantly reduced the luciferase reporter activity in the pGL3-HER23'UTR transfected cells, compared to pGL3-mut HER-3'UTR, whereas the luciferase activity was not affected by the premiR-control ( $\mathrm{P}<0.05$, Fig. 4D).

miR-331-3p exerts its function by targeting HER2. HER2 plays an important role in cell proliferation and cell survival during the development of cancer (30). The results of RT-qPCR in our study showed that HER2-siRNA significantly downregulated the protein expression level of HER2 in the HCT-116 cells $(\mathrm{P}<0.05$, Fig. 5A). HCT-116 cells transfected with AS-miR-331-3p exhibited significantly increased cell proliferation, while cells transfected with HER2-siRNA and co-transfected with AS-miR-331-3p showed significantly decreased proliferation compared with the control. However, there was no statistical difference between cells transfected with both AS-miR-331-3p and HER2-siRNA and treatment with HER2-siRNA alone $(\mathrm{P}<0.05$, Fig. 5B). The results of the FITC-Annexin $\mathrm{V}$ assay showed that premiR-331-3p reduced apoptosis, while HER2-siRNA and the combination of HER2-siRNA and pre-miR-331-3p promoted apoptosis $(\mathrm{P}<0.05$, Fig. 5C).

miR-331-3p suppresses the PI3K/Akt and ERK1/2 signaling pathways. Since HER2 plays important roles in mediating activation of the PI3K/Akt and ERK1/2 pathways $(31,32)$, we aimed to ascertain whether miR-331-3p triggers suppression of HER2, caused by the regulation of PI3K/Akt and ERK1/2 signaling in human CRC cells. As expected, transfection of pre-miR-331-3p significantly inhibited the ratios of the levels of phosphorylated to total protein (arbitrary units) of Akt and ERK1/2, which were both increased by AS-miR-331-3p. HER2-siRNA significantly decreased the arbitrary units of p-Akt and p-ERK1/2. After treatment with HER2-siRNA, the activating effect of AS-miR-331-3p on the arbitrary units of 

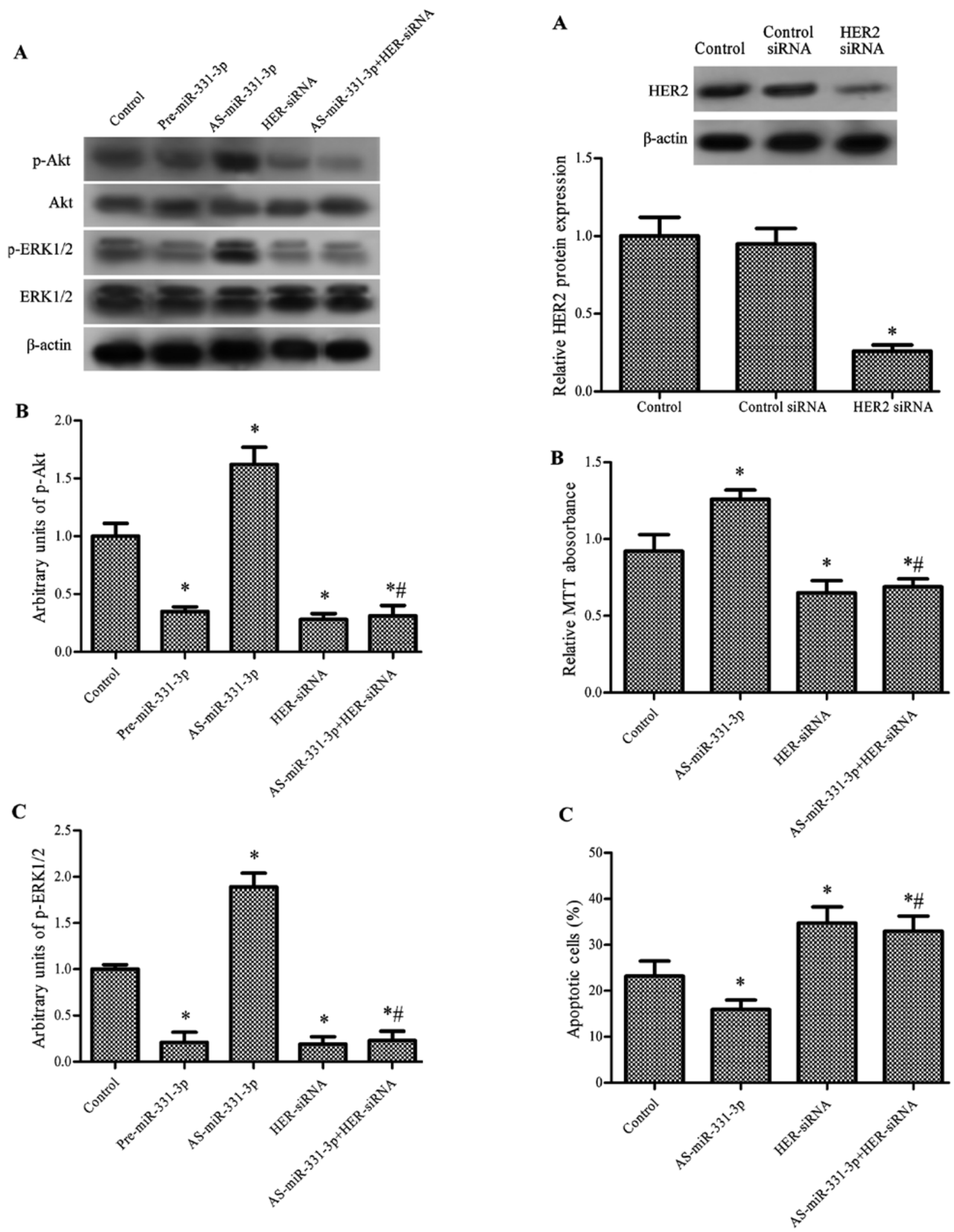

Figure 5. miR-331-3p inhibits cell proliferation and promotes apoptosis by targeting HER2. (A) Western blot analysis of HER2 protein levels in the HCT-116 cells transfected with control siRNA or HER2-siRNA. (B) Cell proliferation was detected by MTT assay in the HCT-116 cells transfected with AS-miR-331-3p or HER2-siRNA or AS-miR-331-3p and HER2-siRNA. (C) Apotosis was detected by Annexin V-FITC assay in the HCT-116 cells transfected with AS-miR-331-3p or HER2-siRNA or AS-miR-331-3p and HER2-siRNA. Bars in the histograms represent the mean \pm standard deviation of three independent experiments. ${ }^{*} \mathrm{P}<0.05$ vs. the control, ${ }^{\#} \mathrm{P}<0.05$ vs. AS-pre-miR-331-3p.

Figure 6. miR-331-3p suppresses the PI3K/Akt and ERK1/2 signaling pathways. (A) Image of western blot analysis of total Akt, p-Akt, total ERK1/2 and $\mathrm{p}$-ERK1/2 protein levels in the HCT-116 cells transfected with pre-miR331-3p, AS-miR-331-3p, HER2-siRNA or AS-miR-331-3p and HER2-siRNA. (B) Quantitative arbitrary units of intensity relative to the control value of p-Akt. (C) Quantitative arbitrary units of intensity relative to the control value of ERK1/2 were normalized to $\beta$-actin. Bars in the histograms represent the mean \pm standard deviation of three independent experiments. ${ }^{*} \mathrm{P}<0.05$ vs. control, ${ }^{,} \mathrm{P}<0.05$ vs. AS-pre-miR-331-3p. 
p-Akt and p-ERK1/2 was abolished and the arbitrary units of p-Akt and p-ERK1/2 were significantly lower than these values in the control $(\mathrm{P}<0.05$, Fig. 6$)$.

\section{Discussion}

In the present study, we provide evidence that miR-331-3p is downregulated in CRC tissues and cells. Overexpression of miR-331-3p plays an important role in inhibiting proliferation and promoting apoptosis by inhibiting the expression of HER2 and deactivation of the PI3K/Akt and ERK1/2 signaling pathways in CRC cells. These results suggest an important role of miR-331-3p in regulating tumor progression of CRC.

miRNAs function as oncogenes or tumor suppressors in carcinogenesis, and dysregulation of these miRNAs is believed to be a common feature of human cancers (33). miRNAs are generally overexpressed or suppressed in tumor tissues, compared with corresponding healthy tissues $(34,35)$. Increasing evidence has demonstrated that miRNAs are crucial regulators of the progression of cancer, with potential use in cancer diagnosis, prognosis, prediction, and therapy $(9,36)$. miR-331-3p is regarded as a cancer-associated miRNA as studies have reported its correlation with prostate cancer, hepatocellular carcinoma, and gastric cancer $(15,16,37)$. In a study by Chang et al (38) on hepatocellular carcinoma, miR-331-3p was overexpressed in human hepatocellular carcinoma tissues and was found to be correlated with poor long-term survival. These authors also demonstrated that miR-331-3p can promote the proliferation and metastasis of hepatocellular carcinoma. Based on these results, they suggested that miR-331-3p may be a potential prognostic biomarker and a novel therapeutic target. Our study found that miR-331-3p was suppressed both in CRC tissues and cells compared with healthy colorectal tissues and cells, which is consistent with the research of Guo et al (16) who found that miR-331-3p was downregulated in gastric cancer cell lines. To further understand the role of miR-331-3p in CRC, the miR-331-3p precursor or miRNA inhibitor of miR331-3p was used to upregulate or downregulate the expression of miR-331-3p, and then cell proliferation and apoptosis were assessed. The results showed that overexpression of miR331-3p significantly inhibited cell proliferation and promoted apoptosis, suggested a crucial role of miR-331-3p in the development of CRC. Together with these results, we confer that the role of miR-331-3p is inconsistent in different types of cancers.

HER2 is overexpressed in a high percentage of CRC cell lines and has been shown to play an oncogenic role in human tumors (39). HER2 can activate signaling pathways including ERK1/2, STAT3, mTOR and Akt, which play important roles in cell proliferation and survival $(31,40,41)$, and therefore is crucial to the development of cancer. Ross and McKenna (42) showed that overexpression of HER2 is associated with approximately one fourth of all gastrointestinal tract malignancies. Li et al (29) found that the expression level of HER2 was elevated in seven out of eight CRC cell lines. Our study measured the expression level of HER 2 in six frequently used CRC cell lines, and all showed upregulated expression of HER2, which suggests that HER2 may be an important factor in the development of CRC.

miRNAs regulate a variety of cellular pathways through targeting the expression of multiple target genes (6). Several lines of evidence indicate that E2F1 and neuropilin-2 are miR-331-3p targets $(15,16)$. In the present study, we showed that miR-331-3p regulated cell proliferation by inhibiting the expression of HER2 in CRC, which is consistent with a study by Epis et al (18), who also concluded that miR-331-3p has the capacity to regulate the development and progression of prostate cancer cells by targeting HER2. PI3K/Akt and ERK1/2 are two important downstream pathways of HER2, and link HER2 to its biological functions (43). While in the study by Epis et al, only the PI3K/Akt signaling pathway was discussed, our study focused on both the PI3K/Akt and ERK1/2 signaling pathways. The results showed that Akt and ERK1/2 were both deactivated by HER2-siRNA and pre-miR-331-3p, and were activated by AS-miR-331-3p. Further research found that the activity of Akt and ERK1/2 were downregulated by the combined action of HER2 and miR-331-3p. PI3K/Akt and ERK1/2 are crucial pathways in regulating cell survival in cancer. These results, taken together, showed that miR-331-3p inhibits proliferation and promotes apoptosis by suppressing HER 2 and deactivating the PI3K/Akt and ERK1/2 signaling pathways.

In conclusion, our study demonstrated that miR-331-3p is suppressed in CRC and overexpression of miR-331-3p inhibits cell proliferation and induces apoptosis by targeting HER2 via activation of the PI3K/Akt and ERK1/2 signaling pathways. Our findings suggest that miR-331-3p plays an important role in the development and progression of CRC.

\section{Acknowledgements}

The present study was supported by grants from the Key Science and Technology Program of Shaanxi Province (no. 2013k12-03-14) and Science and Technology Plan Projects of Xian [SF1203(2)].

\section{References}

1. Siegel R, Desantis C and Jemal A: Colorectal cancer statistics, 2014. CA Cancer J Clin 64: 104-117, 2014.

2. Torre LA, Bray F, Siegel RL, Ferlay J, Lortet-Tieulent J and Jemal A: Global cancer statistics, 2012. CA Cancer J Clin 65: 87-108, 2015.

3. Ferrari P, Jenab M, Norat T, Moskal A, Slimani N, Olsen A, Tjønneland A, Overvad K, Jensen MK, Boutron-Ruault MC, et al: Lifetime and baseline alcohol intake and risk of colon and rectal cancers in the European prospective investigation into cancer and nutrition (EPIC). Int J Cancer 121: 2065-2072, 2007.

4. Edwards BK, Ward E, Kohler BA, Eheman C, Zauber AG, Anderson RN, Jemal A, Schymura MJ, Lansdorp-Vogelaar I, Seeff LC, et al: Annual report to the nation on the status of cancer, 1975-2006, featuring colorectal cancer trends and impact of interventions (risk factors, screening, and treatment) to reduce future rates. Cancer 116: 544-573, 2010.

5. Bosetti C, Levi F, Rosato V, Bertuccio P, Lucchini F, Negri E and La Vecchia C: Recent trends in colorectal cancer mortality in Europe. Int J Cancer 129: 180-191, 2011.

6. Bartel DP: MicroRNAs: Genomics, biogenesis, mechanism, and function. Cell 116: 281-297, 2004.

7. Calin GA, Dumitru CD, Shimizu M, Bichi R, Zupo S, Noch E, Aldler H, Rattan S, Keating M, Rai K, et al: Frequent deletions and down-regulation of micro-RNA genes miR15 and miR16 at 13q14 in chronic lymphocytic leukemia. Proc Natl Acad Sci USA 99: 15524-15529, 2002.

8. Calin GA, Sevignani C, Dumitru CD, Hyslop T, Noch E, Yendamuri S, Shimizu M, Rattan S, Bullrich F, Negrini M, et al: Human microRNA genes are frequently located at fragile sites and genomic regions involved in cancers. Proc Natl Acad Sci USA 101: 2999-3004, 2004. 
9. Croce CM: Causes and consequences of microRNA dysregulation in cancer. Nat Rev Genet 10: 704-714, 2009.

10. Singh SR and Rameshwar P (eds): MicroRNA in Development and in the Progression of Cancer. Springer, New York, NY, 2014.

11. Wang S, Xiang J, Li Z, Lu S, Hu J, Gao X, Yu L, Wang L, Wang J, $\mathrm{Wu} \mathrm{Y}$, et al: A plasma microRNA panel for early detection of colorectal cancer. Int J Cancer 136: 152-161, 2015.

12. Wang L, Tang H, Thayanithy V, Subramanian S, Oberg AL, Cunningham JM, Cerhan JR, Steer CJ and Thibodeau SN: Gene networks and microRNAs implicated in aggressive prostate cancer. Cancer Res 69: 9490-9497, 2009.

13. Zanette DL, Rivadavia F, Molfetta GA, Barbuzano FG, Proto-Siqueira R, Silva-Jr WA, Falcão RP and Zago MA: miRNA expression profiles in chronic lymphocytic and acute lymphocytic leukemia. Braz J Med Biol Res 40: 1435-1440, 2007.

14. Nymark P, Guled M, Borze I, Faisal A, Lahti L, Salmenkivi K, Kettunen E, Anttila S and Knuutila S: Integrative analysis of microRNA, mRNA and aCGH data reveals asbestos- and histology-related changes in lung cancer. Genes Chromosomes Cancer 50: 585-597, 2011.

15. Epis MR, Giles KM, Candy PA, Webster RJ and Leedman PJ: miR-331-3p regulates expression of neuropilin-2 in glioblastoma. J Neurooncol 116: 67-75, 2014

16. Guo X, Guo L, Ji J, Zhang J, Zhang J, Chen X, Cai Q, Li J, Gu Q, Liu B, et al: miRNA-331-3p directly targets E2F1 and induces growth arrest in human gastric cancer. Biochem Biophys Res Commun 398: 1-6, 2010.

17. Epis MR, Giles KM, Kalinowski FC, Barker A, Cohen RJ and Leedman PJ: Regulation of expression of deoxyhypusine hydroxylase $(\mathrm{DOHH})$, the enzyme that catalyzes the activation of eIF5A, by miR-331-3p and miR-642-5p in prostate cancer cells. J Biol Chem 287: 35251-35259, 2012.

18. Epis MR, Giles KM, Barker A, Kendrick TS and Leedman PJ: miR-331-3p regulates ERBB-2 expression and androgen receptor signaling in prostate cancer. J Biol Chem 284: 24696-24704, 2009.

19. Kruser TJ and Wheeler DL: Mechanisms of resistance to HER family targeting antibodies. Exp Cell Res 316: 1083-1100, 2010.

20. Mass RD: The HER receptor family: A rich target for therapeutic development. Int J Radiat Oncol Biol Phys 58: 932-940, 2004.

21. Holbro T and Hynes NE: ErbB receptors: Directing key signaling networks throughout life. Annu Rev Pharmacol Toxicol 44: 195-217, 2004

22. Marmor MD, Skaria KB and Yarden Y: Signal transduction and oncogenesis by ErbB/HER receptors. Int J Radiat Oncol Biol Phys 58: 903-913, 2004.

23. Slamon DJ, Clark GM, Wong SG, Levin WJ, Ullrich A and McGuire WL: Human breast cancer: Correlation of relapse and survival with amplification of the HER-2/neu oncogene. Science 235: 177-182, 1987

24. Yu D and Hung MC: Overexpression of ErbB2 in cancer and ErbB2-targeting strategies. Oncogene 19: 6115-6121, 2000.

25. Tan M, Yao J and Yu D: Overexpression of the c-erbB-2 gene enhanced intrinsic metastasis potential in human breast cancer cells without increasing their transformation abilities. Cancer Res 57: 1199-1205, 1997.
26. Yu D, Jing T, Liu B, Yao J, Tan M, McDonnell TJ and Hung MC: Overexpression of ErbB2 blocks Taxol-induced apoptosis by upregulation of $\mathrm{p} 21^{\mathrm{Cip} 1}$, which inhibits $\mathrm{p} 34^{\mathrm{Cdc} 2}$ kinase. Mol Cell 2: 581-591, 1998.

27. Blok EJ, Kuppen PJ, van Leeuwen JE and Sier CF: Cytoplasmic overexpression of HER2: A key factor in colorectal cancer. Clin Med Insights Oncol 7: 41-51, 2013.

28. Schmittgen TD and Livak KJ: Analyzing real-time PCR data by the comparative C(T) method. Nat Protoc 3: 1101-1108, 2008

29. Li SS, Buchbinder E, Wu L, Bjorge JD, Fujita DJ and Zhu S: EGFR and HER2 levels are frequently elevated in colon cancer cells. Discoveries Rep 1: e1, 2014.

30. Hynes NE and MacDonald G: ErbB receptors and signaling pathways in cancer. Curr Opin Cell Biol 21: 177-184, 2009.

31. Nahta R and O'Regan RM: Evolving strategies for overcoming resistance to HER2-directed therapy: Targeting the PI3K/Akt/ mTOR pathway. Clin Breast Cancer 10 (Suppl 3): S72-S78, 2010.

32. Citri A and Yarden Y: EGF-ERBB signalling: Towards the systems level. Nat Rev Mol Cell Biol 7: 505-516, 2006.

33. Chen CZ: MicroRNAs as oncogenes and tumor suppressors. N Engl J Med 353: 1768-1771, 2005.

34. Motoyama K, Inoue H, Takatsuno Y, Tanaka F, Mimori K, Uetake H, Sugihara K and Mori M: Over- and under-expressed microRNAs in human colorectal cancer.Int J Oncol 34: 1069-1075, 2009.

35. Lu J, Getz G, Miska EA, Alvarez-Saavedra E, Lamb J, Peck D, Sweet-Cordero A, Ebert BL, Mak RH, Ferrando AA, et al: MicroRNA expression profiles classify human cancers. Nature 435: 834-838, 2005.

36. Calin GA and Croce CM: MicroRNA signatures in human cancers. Nat Rev Cancer 6: 857-866, 2006.

37. Epis MR, Barker A, Giles KM, Beveridge DJ and Leedman PJ: The RNA-binding protein HuR opposes the repression of ERBB-2 gene expression by microRNA miR-331-3p in prostate cancer cells. J Biol Chem 286: 41442-41454, 2011.

38. Chang RM, Yang $\mathrm{H}$, Fang $\mathrm{F}, \mathrm{Xu}$ JF and Yang LY: MicroRNA-331-3p promotes proliferation and metastasis of hepatocellular carcinoma by targeting $\mathrm{PH}$ domain and leucine-rich repeat protein phosphatase. Hepatology 60: 1251-1263, 2014.

39. Kafi SG, Lari S and Nassiri G: HER $2 /$ neu expression in colon adenocarcinoma and its correlation with clinicopathologic variables. IJBMS 9: 64-69, 2006.

40. Kim SY, Kim HP, Kim YJ, Oh Y, Im SA, Lee D, Jong HS, Kim TY and Bang YJ: Trastuzumab inhibits the growth of human gastric cancer cell lines with HER2 amplification synergistically with cisplatin. Int J Oncol 32: 89-95, 2008

41. Mebratu Y and Tesfaigzi Y: How ERK1/2 activation controls cell proliferation and cell death: Is subcellular localization the answer? Cell Cycle 8: 1168-1175, 2009.

42. Ross JS and McKenna BJ: The HER-2/neu oncogene in tumors of the gastrointestinal tract. Cancer Invest 19: 554-568, 2001.

43. Yarden Y and Sliwkowski MX: Untangling the ErbB signalling network. Nat Rev Mol Cell Biol 2: 127-137, 2001. 